\title{
Prospective Study
}

\section{e Fluoroscopically Guided Thoracic Interlaminar Epidural Injection: A Comparative Epidurography Study Using $2.5 \mathrm{~mL}$ and $5 \mathrm{~mL}$ of Contrast Dye}

JiHee Hong, MD, PhD, and Sung won Jung, MD, PhD

\footnotetext{
From: Keimyung University School of Medicine Republic of Korea

Address Correspondence: JiHee Hong, MD, PhD Department of Anesthesiology and Pain Medicine Keimyung University Dong San Hospital

56, Dal-Sung Ro, Jung gu Dae Gu, 700-712, Korea E-mail:swon13@daum.net

Disclaimer: This work was supported by the National Research Foundation of Korea(NRF) Grant funded by the Korea Government (MSIP) (No.2014R1A5A2010008).Conflict of interest: Each author certifies that he or she, or a member of his or her immediate family, has no commercial association (i.e., consultancies, stock ownership, equity interest, patent/licensing arrangements, etc.) that might pose a conflict of interest in connection with the submitted manuscript.

Manuscript received: 01-17-2016

Revised manuscript received: 02-25-2016

Accepted for publication: 03-11-2016

Free full manuscript: www.pain physicianjournal.com
}

Background: Thoracic epidural anesthesia (TEA) is frequently used to maintain intraoperative analgesia. After injecting the initial bolus dose of epidural local anesthetics (LA), intermittent injection of LA through an epidural catheter is required to maintain the intraoperative analgesia. For intermittent epidural administration, usually $2-5 \mathrm{~mL}$ of LA has been used. However, no studies have suggested an optimal volume of LA of TEA for intermittent epidural administration of TEA.

Objective: We focused on identifying an optimal volume of LA of TEA using epidurography of the thoracic level with 2 different volumes of contrast dye.

Study Design: Prospective, randomized study.

Setting: An interventional pain management practice in South Korea.

Methods: After Institutional Review Board approval, 70 patients undergoing thoracic epidural catheterization for upper abdominal and thoracic surgery were randomly assigned to one of the 2 contrast dye volume groups of 35 patients each $(A, 2.5 \mathrm{~mL}$ and $B, 5.0 \mathrm{~mL})$. Epidurography was evaluated to confirm how many spinal segments were covered by contrast dye. The spreads in the cephalad and caudad directions were also evaluated.

Results: The total number of vertebral segments evaluated by contrast dye were $7.5 \pm 2.0$, and $8.4 \pm 2.6$, respectively in groups $A$ and $B$. The number of patients who showed contrast dye spread of more than 5 vertebral segments was 34/35 (97\%) in both groups. Group B resulted in higher contrast dye distribution in the cephalad direction compared to group A (T2.6 vs. T3.6 ).

Limitations: We used a test dose of contrast dye to confirm the contrast was in epidural space, not intrathecal or vascular, before injection of the main dose of contrast dye. The present study did not include the volume of test dose.

Conclusion: The volume of $2.5 \mathrm{~mL}$ for intermittent epidural administration would be enough for the analgesic effect of upper abdominal and thoracic surgery while avoiding excessive upper thoracic and cervical spread.

Key words: Thoracic epidural anesthesia, intermittent epidural administration, optimal volume, epidurography, cephalad, caudad, analgesic effect

Pain Physician 2016; 19:E1013-E1018 horacic epidural anesthesia (TEA) is frequently used to maintain intraoperative analgesia and also used for postoperative pain management. Epidural administration of local anesthetics (LA) with or without opioids is known to provide better quality postoperative pain relief compared to systemic administration of an opioid $(1,2)$. In addition, epidural anesthesia showed more favorable effects on coagulation and homeostasis due to the attenuating effect on central sympathetic stimulation (3). Epidural 
anesthesia has also been suggested as a safe method in reducing the incidence of venous thrombosis and cardiac events in patients undergoing total hip replacement and vascular surgery, respectively $(4,5)$. Thoracic epidural anesthesia can also be applied to upper abdominal and thoracic surgery including lobectomy or thoracotomy, and it showed the protective effect on pulmonary complications (2).

The level of sensory blockade after epidural injection of LA differs considerably between individuals, and various factors that might affect this distribution still remain controversial. Visser et al (6) suggested that total mass of LA appears to be the most important factor in determining the level of sensory, sympathetic, and motor blockade. However, increasing the total dose of LA for a linear relationship in the level of sensory blockade has been questioned (6).

In our clinical practice, after injecting the initial bolus dose of epidural LA, intermittent injection of LA through an epidural catheter is required to maintain the intraoperative analgesia. For the injection of this intermittent epidural administration, usually $2-5 \mathrm{~mL}$ of LA has been used. However, no studies have suggested an optimal volume of LA that could be used for an intermittent epidural administration of TEA.

According to the study by Yokoyama et al (7), epidural spread of contrast medium correlated well with the spread of LA, therefore, epidurography could predict the dermatomal distribution of sensory blockade.

In this study, we focused on identifying an optimal volume of LA of TEA using epidurography of the thoracic level with different volumes of contrast dye.

The primary endpoint of this study was to evaluate, after fluoroscopically guided thoracic epidural catheter injection, how many spinal segments could be covered with different volumes of contrast medium.

\section{Methods}

This study was approved by the Institutional Review Board (IRB) of our institution. We explained the benefits, risks, and goals of this study to the patients and obtained written informed consent from all patients. From March 2015 to November 2015, 72 patients who received fluoroscopically guided thoracic interlaminar epidural catheter insertion were enrolled in this study. The patients who were scheduled to receive elective upper abdominal surgery including laparoscopic gastrectomy, esophagectomy, liver lobectomy, and thoracic surgery were inserted with thoracic epidural catheter by fluoroscopic guidance one day before surgery for intraoperative and postoperative pain management.

We included those patients who had stopped taking anticoagulants for the required time before thoracic epidural catheter insertion. We excluded those patients with pregnancy, laboratory findings suggesting infection, inflammation or coagulopathy, and allergy to contrast dye or LA. Those patients who received spine surgery or patients with ankylosing spondylitis or other spine disease at the thoracic level were excluded.

Two patients were excluded due to refusal to participate in this study among the initial 72 patients. Finally, 70 patients were enrolled and 70 cases of thoracic epidural catheter insertion were analyzed.

Seventy patients were randomly assigned into 2 groups according to the amount of contrast dye used (Group A, $2.5 \mathrm{~mL}$ and Group B, $5 \mathrm{~mL}$ ). All catheter insertions were performed using the paramedian approach at the T10-11 level. Patients were placed in the prone position and draped in a sterile fashion. Anteroposterior view (AP) was obtained to confirm the T10-11 interlaminar space. After skin infiltration with $1 \mathrm{~mL}$ of $1 \%$ lidocaine, an $18 \mathrm{G}$ Tuohy needle was inserted toward the paramedian interlaminar site of T10-11, and advanced slowly into midline using loss of resistance with air. When loss of resistance was felt, the lateral fluoroscopic view was obtained to confirm that the needle was positioned within the posterior border of the spinal canal. After checking the needle position, $1 \mathrm{~mL}$ of contrast dye was injected for confirming the epidural injection with an AP and lateral fluoroscopic image.

\section{Primary Outcome Measure: Analysis of Contrast Flow}

In cases of successful epidural injection, an epidural catheter was inserted through a Tuohy needle, and $2.5 \mathrm{~mL}$ or $5 \mathrm{~mL}$ of contrast dye was injected through the epidural catheter according to the assigned study group. A physician who was blinded to the assigned study group evaluated the epidurography. First, the position of the catheter tip was assessed and the number of spinal segments covered by contrast dye was evaluated. We observed the cephalad and caudal end of the spinal segment covered by contrast dye to evaluate the total spinal segment.

The extent of contrast dye spread was assessed by evaluating the upper and lower endplates of the vertebra on the lateral view (Fig. 1A). However, the first to third thoracic vertebra were very difficult to evaluate for the contrast spread pattern on the lateral view, 


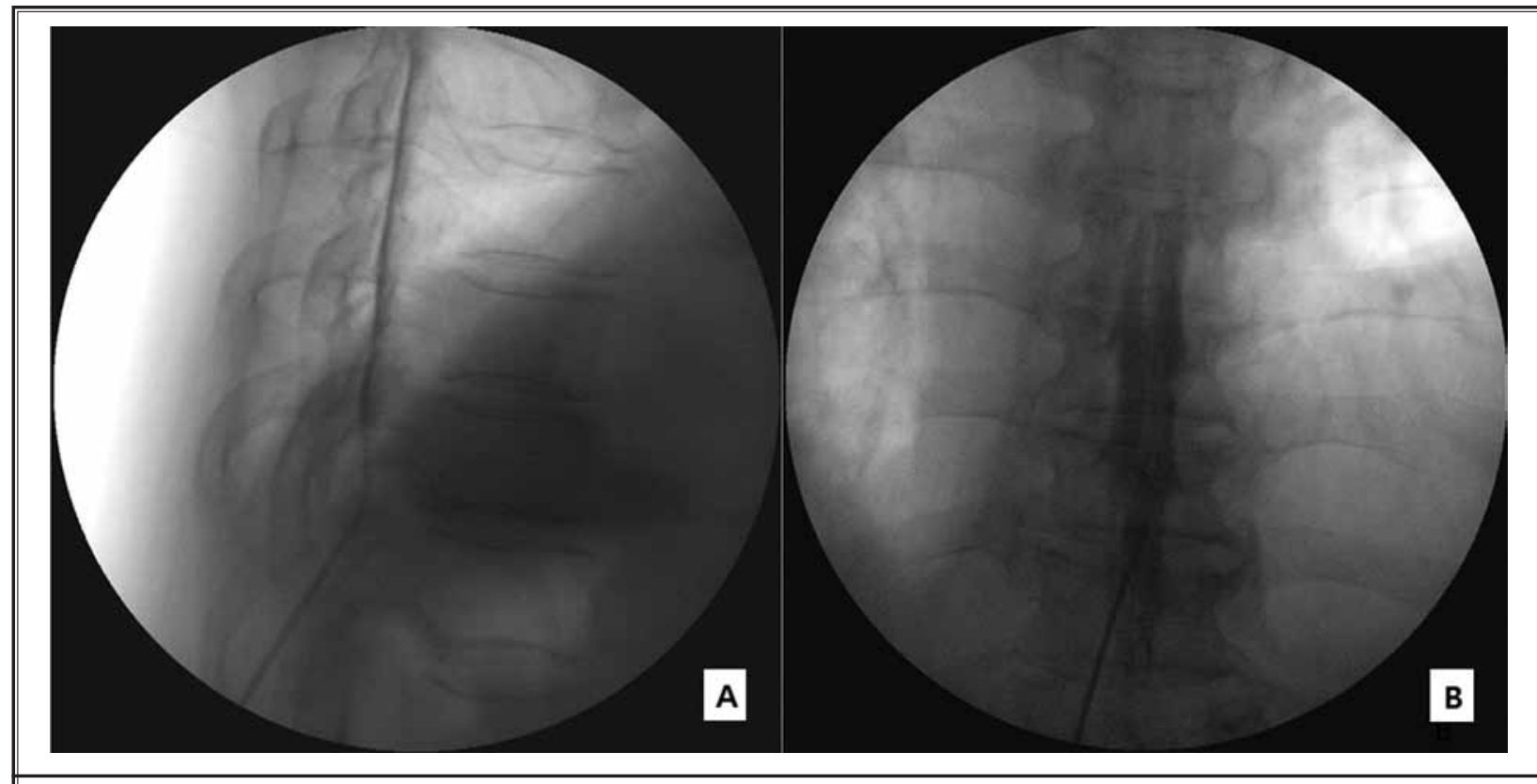

Fig. 1. The extent of contrast dye spread was assessed by evaluating the upper and lower endplates of the vertebra on the lateral view (A) and unilateral or bilateral epidural spread by checking the anteroposterior fluoroscopic image (B).

therefore, we assessed the AP fluoroscopic image in such cases.

Included in the counting were the spinal segments covered by contrast dye on the lateral view for more than half of their vertebral body height, and excluded were the segments with the contrast dye spread covering less than half of their vertebral body height. We also assessed the unilateral or bilateral epidural spread by checking the AP fluoroscopic image (Fig. 1B).

All patients who finished their epidurography study were sent to their hospital room, and the next day, upper abdominal or thoracic surgery was performed with general anesthesia combined with TEA.

\section{Statistical Analysis}

As there was no previous data for contrast flow in TEA according to different volumes, we first performed an initial preliminary study for an estimate of the proper sample size. According to our preliminary study, $60 \%$ and $95 \%$ of patients of group A and B, respectively, resulted in contrast dye spread of more than 7 segments in epidurography findings. It is suggested that 10 dermatomal blockade of TEA without general anesthesia is needed for upper abdominal or thoracic surgery (8). Therefore, we thought that 7 segments of sensory blockade would be enough to maintain intraoperative analgesia when combined with general anesthesia. As- suming the difference of incidence rate of contrast dye spread of more than 7 segments as 0.35 and an $\alpha$ error level of 0.05 , a $\beta$ error level of $0.02,35$ patients of TEA were required in each group with a power of $80 \%$.

\section{Results}

Among 72 patients selected for this study, 2 patients refused to participate in this study. Therefore, 70 patients were enrolled and 70 cases of thoracic epidural catheter insertion were analyzed. There were no serious adverse events related to the procedure. There were no statistical differences in age, gender, body mass index, and type of surgery for TEA between the 2 groups. Almost half of the included patients received lung lobectomy due to lung cancer (Table 1).

The total number of vertebral segments marked by contrast dye spread were $7.5 \pm 2.0$ in group $A(2.5 \mathrm{~mL})$ and $8.4 \pm 2.6$ in group $B(5 \mathrm{~mL})$, and the spread was in both cephalad and caudad directions. There were no significant differences between the 2 groups with respect to the number of vertebral segments showing dye spread. All patients in both groups showed bilateral contrast dye distribution evenly. The contrast dye spread in the cephalad direction showed more extensive distribution than the caudad direction in groups $A$ and $B$ and it demonstrated statistical significance $(P<0.005)$. Group B resulted in higher contrast dye 
distribution in the cephalad direction compared to group A (T2.6 vs. T3.6) and it demonstrated statistical significance $(P<0.05)$. Whereas, contrast dye distribution in the caudad direction was similar between groups $A$ and $B$.

Four patients from groups $A$ and $B$ showed contrast dye spread up to the cervical segment and one of them from group B showed contrast dye spread until the second cervical vertebra.

\section{Discussion}

The distribution of TEA medication in the epidural space depends on the injection site, patient position, age, height, weight, speed of injection, and volume of the injection. Volume is a major factor determining the spread range of an injected medication $(6,9)$. However, few studies have reported the optimal volume of medication for TEA. Contrast dye spread has a clear correlation with the extent of LA blockade, and epidurography can help to predict the distribution of medication (7).

According to our thoracic epidurography study, groups $A(2.5 \mathrm{~mL})$ and $B(5 \mathrm{~mL})$ volumes of contrast dye resulted in

Table 1. Demographic data and type of surgery in the study population.

\begin{tabular}{|l|c|c|}
\hline & Group A (2.5 mL) & Group B (5.0 mL) \\
\hline Age (years) & $61.0 \pm 10.5$ & $59.5 \pm 13.2$ \\
\hline Gender (M/F) & $25 / 10$ & $24 / 11$ \\
\hline Body mass index $\left(\mathrm{kg} / \mathrm{m}^{2}\right)$ & $23.1 \pm 3.0$ & $24.3 \pm 2.5$ \\
\hline \multicolumn{3}{|l|}{ Type of surgery } \\
\hline Laparoscopic Gastrectomy & 9 & 3 \\
\hline Esophagectomy & 0 & 3 \\
\hline Liver lobectomy & 11 & 9 \\
\hline Lung lobectomy & 15 & 20 \\
\hline \hline
\end{tabular}

Values are mean \pm SD or number of patients. There were no significant differences between the 2 groups. total dye spread of $7.5 \pm 2.0$ and $8.4 \pm 2.6$ vertebral segments, respectively. Although total contrast dye spread was similar between the 2 groups, the most upper level of cephalad spread was higher, and more patients showed contrast dye spread up to cervical segments in group $B$.

It has been known that epidural anesthesia does influence pulmonary function due to effects on motor function and sympathetic innervation, although these effects including reductions in vital capacity and forced expiratory volume are insignificant under lumbar and low thoracic epidural anesthesia. However, these reduced pulmonary functions can increase up to $20 \%$ or $30 \%$ of baseline if sensory blockade reaches higher levels of the vertebral column (10).

According to Huang (11), cervical epidural anesthesia with $2 \%$ lidocaine showed reduced lung volumes and capacities resulting from a paralytic effect on the intercostal muscles and diaphragm innervated by the thoracic intercostal and phrenic nerve, respectively. Considering these respiratory complications for high thoracic and cervical epidural anesthesia, excessive cephalad and cervical level distribution of medication needs to be avoided. Also, neck flexion during high TEA resulted in increased cephalad spread of contrast dye (12).

For both groups, the contrast dye spread in the cephalad direction was more extensive than in the caudad direction. This finding was similarly observed in epidurography study of the cervical spine. Regardless of the injected contrast dye volume, all groups showed more extensive distribution in the cephalad direction compared to caudad direction (13). If TEA should be performed at the

Table 2. The mean vertebral segment of contrast flow at the catheter tip.

\begin{tabular}{|l|c|c|}
\hline & Group A (2.5 mL) & Group B (5 mL) \\
\hline Position of catheter tip & T7.9 & T7.1 \\
\hline Number of segments covered with cephalad spread & $4.4 \pm 1.5^{*}$ & $4.8 \pm 2.4^{*}$ \\
\hline Number of segments covered with caudad spread & $3.1 \pm 1.2$ & $3.5 \pm 1.4$ \\
\hline Total number of segments covered with spread & $7.5 \pm 2.0$ & $8.4 \pm 2.6$ \\
\hline Most upper level of cephalad spread & T3.6 & T2.6† \\
\hline Most lower level of caudad spread & T10.9 & T10.6 \\
\hline
\end{tabular}

Values are mean \pm SD. T: thoracic vertebra.

There were no statistical significances in cephalad and caudad spread of contrast flow between groups A and B. The contrast spread of cephalad direction was more extensive than the caudad direction. ${ }^{*} P<0.005$. The upper tip level of cephalad spread was higher in group $\mathrm{B}$ com pared to group A. $\dagger P<0.05$ 
middle thoracic vertebral level, it is very difficult technically due to the steep downward angulation of the spinous process. However, in spite of this technical difficulty, TEA at the middle thoracic area is performed to obtain proper sensory blockade reaching the upper thoracic dermatome. We think that if TEA is performed at T10-11 which is easier than the middle thoracic level, sufficient sensory block ranging from the middle to upper thoracic dermatome would be possible considering the fact that contrast dye shows more extensive cephalad spread than caudad spread.

During lumbar epidural anesthesia, we may encounter insufficient sensory blockade of one leg compared to another leg, although this is infrequent. In such cases, we can suspect unilateral distribution of medication, either to the right or left side. In our study, both groups showed even and bilateral distribution of contrast dye regardless of the injected volume.

Our study includes several limitations. We used a small test dose of contrast dye to confirm the contrast was in the epidural space, not the intrathecal or vascular, before injection of the main dose of contrast dye. The present study did not include the exact volume of the test dose.

Also, the first to third thoracic vertebra were assessed on the AP fluoroscopic image due to the difficulty of assessment of the lat-
Table 3. Incidence of constrast dye spread up to cervical and lumbar segments.

\begin{tabular}{|l|l|l|}
\hline & Group A (2.5 mL) & Group B (5 mL) \\
\hline Spread up to cervical segments & $1 / 35$ & $3 / 35$ \\
\hline Spread up to lumbar segments & $3 / 35$ & $2 / 35$ \\
\hline
\end{tabular}

There were no significant differences between group A and B.

eral image. In such cases, the contrast dye spread measures might be slightly different from images obtained from the lateral view.

In summary, when selecting the optimal volume of medication, the range and pattern of spread is important. Therefore, we suggest that the volume of $2.5 \mathrm{~mL}$ for intermittent epidural administration would be sufficient for the analgesic effect for upper abdominal and thoracic surgery while avoiding excessive upper thoracic and cervical spread. In addition, we can expect more stable hemodynamics with less LA toxicity when lesser volume of LA was used.

\section{Conclusion}

When selecting the most optimal volume of medication, considering the range and pattern of spread of the contrast dye is important. Therefore, we suggest that the volume of $2.5 \mathrm{~mL}$ for intermittent epidural administration would be enough for the analgesic effect for upper abdominal and thoracic surgery while avoiding excessive upper thoracic and cervical spread.

\section{Acknowledgement}

We would like to give special thanks to Jina Lee and Eunseok Kim who have contributed making high guality figures.

\section{References}

1. Nishimori $\mathrm{M}$, Low JH, Zheng $\mathrm{H}$, Ballantyne JC. Epidural pain relief versus systemic opioid-based pain relief for abdominal aortic surgery. Cochrane Database Syst Rev 2012; 7:Cdo05059.

2. Popping DM, Elia N, Marret E, Remy C, Tramer MR. Protective effects of epidural analgesia on pulmonary complications after abdominal and thoracic surgery: A meta-analysis. Arch Surg 2008; 143:990-999.

3. Korakianitis O, Daskalou T, Alevizos L, Stamou K, Mavroudis C, latrou C, Vogiatzaki T, Eleftheriadis $\mathrm{S}$, Tentes AA. Lack of significant intraoperative coagulopathy in patients undergoing cytoreductive surgery and hyperthermic intraperitoneal chemotherapy (HIPEC) indicates that epidural anaesthesia is a safe option. Int J Hyperthermia 2015; 31:857-862.

4. Neuman MD, Silber JH, Elkassabany NM, Ludwig JM, Fleisher LA. Comparative effectiveness of regional versus general anesthesia for hip fracture surgery in adults. Anesthesiol 2012; 117:72-92.

5. Beattie WS, Badner NH, Choi P. Epidural analgesia reduces postoperative myocardial infarction: A meta-analysis. Anesth Analg 2001; 93:853-858.

6. Visser WA, Lee RA, Gielen MJ. Factors affecting the distribution of neural blockade by local anesthetics in epidural anesthesia and a comparison of lumbar ver- sus thoracic epidural anesthesia. Anesth Analg 2008; 107:708-721.

7. Yokoyama $M$, Hanazaki $M$, Fujii $H$, Mizobuchi S, Nakatsuka H, Takahashi T, Matsumi M, Takeuchi M, Morita K. Correlation between the distribution of contrast medium and the extent of blockade during epidural anesthesia. Anesthesiol 2004; 100:1504-1510.

8. Iwata Y, Hamai Y, Koyama T. Anesthetic management of nonintubated video assisted thoracosopic surgery using epidural anesthesia and dexmedetomidine in three patients with severe respiratory dysfunction. J Anesth 2016; 13:1-4.

9. Lee SE, Joe HB, Park JH, Yi IK, Choi YH, Han KR, Kim C. Distribution range of 
cervical interlaminar epidural injections: a comparative study with $2.5 \mathrm{~mL}, 5 \mathrm{~mL}$, and $10 \mathrm{~mL}$ of contrast. Pain Physician 2013; 16:155-164.

10. Zheng J, Du L, Liu b. Influence of body mass index and epidural anesthesia on lung function. Anesthesiol 2014; 120:509-510.
11. Huang $\mathrm{CH}$. Effect of cervical epidural blockade with $2 \%$ lidocaine plus epinephrine on respiratory function. Acta Anaesthesiol Taiwan 2007; 45:217-222.

12. Lee CJ, Jeon Y, Lim YJ, Bahk JH, Kim YC, Lee SC, Kim CS. The influence of neck flexion and extension on the distribution of contrast medium in the high thoracic epidural space. Anesth Analg 2007; 104:1583-1586.

13. Kim KS, Shin SS, Kim TS, Jeong CY, Yoon $\mathrm{MH}$, Choi JI. Fluoroscopically guided cervical interlaminar epidural injections using the midline approach: An analysis of epidurography contrast patterns. Anesth Analg 2009; 108:1658-1661. 